\title{
A new method for combining of genetically correlated traits by maximizing of their shared heritability
}

\author{
Gulnara R. Svishcheva \\ Laboratory of recombination and \\ segregation analysis \\ Institute of cytology and genetics \\ Novosibirsk, Russia \\ Elizaveta E. Elgaeva \\ Laboratory of recombination and \\ segregation analysis \\ Institute of cytology and genetics \\ Novosibirsk, Russia
}

\author{
Evgeny S. Tiys \\ Laboratory of glycogenomics \\ Institute of cytology and genetics \\ Novosibirsk, Russia \\ Sodbo Sharapov \\ Laboratory of recombination and \\ segregation analysis \\ Institute of cytology and genetics \\ Novosibirsk, Russia
}

\author{
Sofya G. Feoktistova \\ Kurchatov genomic center \\ Institute of cytology and genetics \\ Novosibirsk, Russia \\ Yakov A. Tsepilov \\ Laboratory of Theoretical and Applied \\ Functional Genomics \\ Novosibirsk State University \\ Novosibirsk, Russia
}

\begin{abstract}
Genetic correlations between phenotypic traits are widely observed phenomena. Many groups of traits have a significant level of shared genetic background. In this work, we suggested a new method for extraction of shared genetic component for genetically correlated phenotypic traits. We applied the method to GWAS results for anthropometric traits.
\end{abstract}

Keywords - shared heredity, anthropometry, GWAS.

\section{Introduction}

In the era of large sample sizes available for genome wide association studies (GWAS), it becomes clear that a remarkable number of human traits and diseases are highly genetically correlated. In other words, many groups of traits have a significant level of shared genetic background. There is a big temptation to be able to study this background thought analysis a new aggregate trait that approximates shared heritability of the studied traits.

\section{Materials and methods}

We propose a new strategy to the SNP-based association analysis of multiple genetically correlated traits. This approach focuses on a linear combination of the traits by maximizing of heritability explained by SNPs associated with all the traits (named as 'shared' SNPs). The first step is to decompose a matrix of genetic correlations between traits into two matrices so that one of them is explained by shared SNPs, while the other represents the genetic correlations formed by the unique genetic background of each trait. We compared several matrix decomposition techniques, including eigen-, CUR-, and NMF- decompositions. Maximum quality was achieved when we used a method that minimizes absolute values of trait-trait correlations explained the unique genetic background. The second step allows us to analytically estimate the linear combination weights of traits by maximizing their shared heritability explained only the shared SNPs. Finally, in the last step, GWAS for analyzing shared heredity is performed as a linear combination of single-traitlevel GWAS summary statistics.

To assess this method we used GWAS data from such widely studied anthropometry traits as weight, BMI, waist circumference and hip circumference. Data for anthropometric traits was downloaded from http://www.nealelab.is/uk-biobank. In the first step, we found coefficients of linear trait combinations that maximizes the shared heredity between them. In the next step, we used the estimated coefficients to construct GWAS summary statistics for shared heredity. The significant threshold was set as $5 \times 10^{-}$ ${ }^{8}$. The SNPs were considered from one locus if they were located within $1000 \mathrm{~kb}$ from top associated SNP. Using Fisher's exact test we found significant enrichment of top significantly associated SNPs with combined trait among top SNPs associated with all traits.

\section{Results}

We tested our method on simulated data using different scenarios. In each scenario, we randomly generated the correlation matrix component explained by the non-shared SNPs, using parameters $w \in(0,1)$ (the contribution of each trait to shared heritability) and $e \in(0,1)$ (variance of random variables that determine correlations caused by a unique genetic background). Besides, we formed the matrix explained by the shared SNPs, using $w$. In each scenario, 10,000 repeats were performed. For each repeat, we tested the generated matrices for compliance with the definition of a valid correlation matrix. For small and moderate $e$, and moderate or large $w$, the mean difference between the observed and expected $w$ was very small $(0.0041 \pm 0.0008)$.

In analysis of real data, we found 729 loci significantly associated with common genetic component (shared heredity) for the anthropometric traits. Among them, 28 loci are not significantly associated with any of the original traits and may be considered as new.

We estimated the enrichment of SH loci among the loci that are significantly associated with all individual traits versus loci that were not significant with all individual traits at the same time. The significance of enrichment was $p=2.3 \times 10^{-32}$. We plan to apply this method for such genetically correlated traits as plasma lipid levels and several psychiatric disorders.

\section{ACKNOWLEDGMENT}

The work of G.S. was supported by the RFBR grant (2004-00464). The work of S.F., E.T. and SSh was funded by the Russian Science Foundation grant number 19-15-00115. The work of EEE and YAT was supported by the Russian Foundation for Basic Research (project 19-015-00151). 